\title{
A Case of Poncet's Disease: Tuberculous Rheumatism
}

\author{
Esra ERKOL INAL, Dilek KESKIN, Hatice BODUR \\ Department of Physical Medicine and Rehabilitation, Ankara Numune Training and Research Hospital, Ankara, Turkey
}

\begin{abstract}
Poncet's disease (PD), a reactive caused by Mycobacterium tuberculosis, is a rare condition. In this paper, we report the case of a 57-year-old woman with PD who referred to hospital with recurrent polyarthritis. She had been using sulphasalazine $3 \mathrm{~g} /$ day, prednisolone $10 \mathrm{mg} /$ day, and naproxen sodium $1100 \mathrm{mg} /$ day because she was diagnosed with rheumatoid arthritis. However, this medication did not provide sufficient relief of symptoms. Then, methotrexate $10 \mathrm{mg} /$ week was added to her medical therapy. There was no regression of her symptoms during her follow up. She was finally diagnosed with PD; her antirheumatic drugs were stopped, and four-drug antituberculosis therapy was started. At the sixth month visit after discharge, her complaints and clinical symptoms completely disappeared and her laboratory tests were normalized.
\end{abstract}

Keywords: Poncet's disease, tuberculous rheumatism, polyarthritis, tuberculosis

\section{Introduction}

Poncet's disease (PD) is a reactive polyarthritis where no mycobacterial involvement can be detected after acute tuberculosis (1-3). In this paper, we reported a case of PD that occurred with recurrent polyarthritis.

\section{Case Report}

A 57-year-old woman was admitted to our hospital with symptoms of recurrent polyarthritis. She complained of morning stiffness lasting for 1-1.5 h. She had oligoarthritis on her ankles and metatarsophalangeal (MTP) joints eight months ago, and since then, she was using sulphasalazine $3 \mathrm{~g} /$ day, prednisolone $10 \mathrm{mg} /$ day, and naproxen sodium $1100 \mathrm{mg} /$ day. However, these medications did not provide sufficient relief from of the symptoms. Her laboratory tests were as follows: hemoglobin $(\mathrm{Hb})$, $10.5 \mathrm{~g} / \mathrm{dL}$; hematocrit (Htc), 31.7\%; white blood cell (WBC) count, $5100 / \mathrm{mm}^{3}$; thrombocyte, $205000 / \mathrm{mm}^{3}$; erythrocyte sed- imentation rate (ESR), $51 \mathrm{~mm} / \mathrm{h}$; C-reactive protein (CRP), 73.2 $\mathrm{mg} / \mathrm{L}$; and rheumatoid factor (RF), $17.4 \mathrm{IU} / \mathrm{mL}$. She had normal liver and kidney function tests, and her urinalysis was also normal. Urine and throat cultures were sterile after incubation. Her chest and hand radiogram were normal (Figure 1,2). On physical examination of her wrists, ankles, proximal interphalangeal, metacarpophalangeal and MTP joints were tender on palpation, and her left fourth PIF joint was swollen (Figure 3). This symmetric polyarthritis that is prominent especially on the upper limbs led us to a diagnosis of rheumatoid arthritis (RA), and methotrexate $10 \mathrm{mg} /$ week was added to her medical therapy. At the first month visit, the methotrexate dosage was increased to 15 $\mathrm{mg} /$ week but, there was no regression of her symptoms during her follow-up. At the third month control, her laboratory tests showed a hgb level of $11.9 \mathrm{~g} / \mathrm{dL}$, Htc value of $36.3 \%$, a WBC count of $12400 / \mathrm{mm}^{3}$, thrombocyte count of $447000 / \mathrm{mm}^{3}$, ESR of $77 \mathrm{~mm} / \mathrm{h}$, and CRP level of $146 \mathrm{mg} / \mathrm{L}$. She was hospitalized in order to find out whether it was reactive arthritis or a reflection 


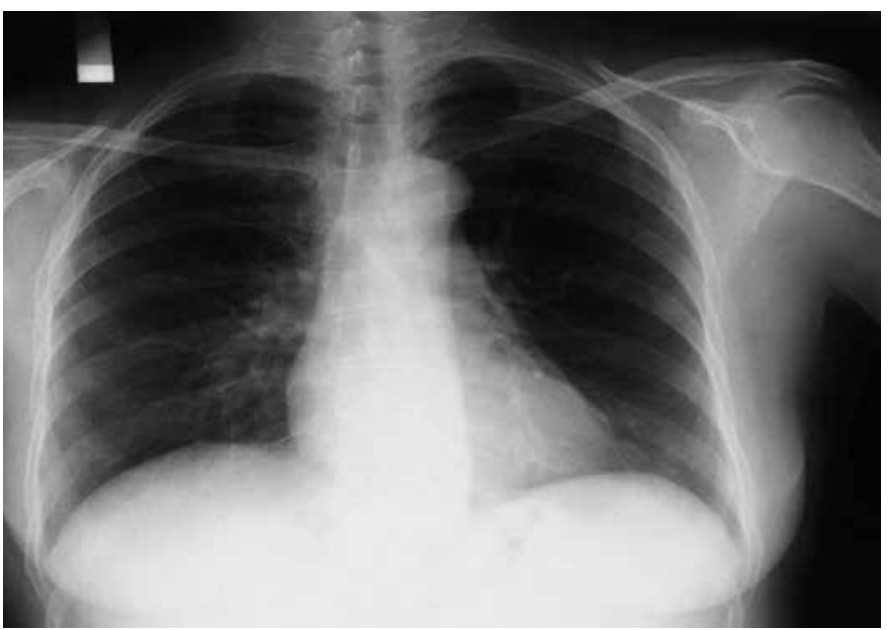

Figure 1. Normal postero-anterior chest radiogram

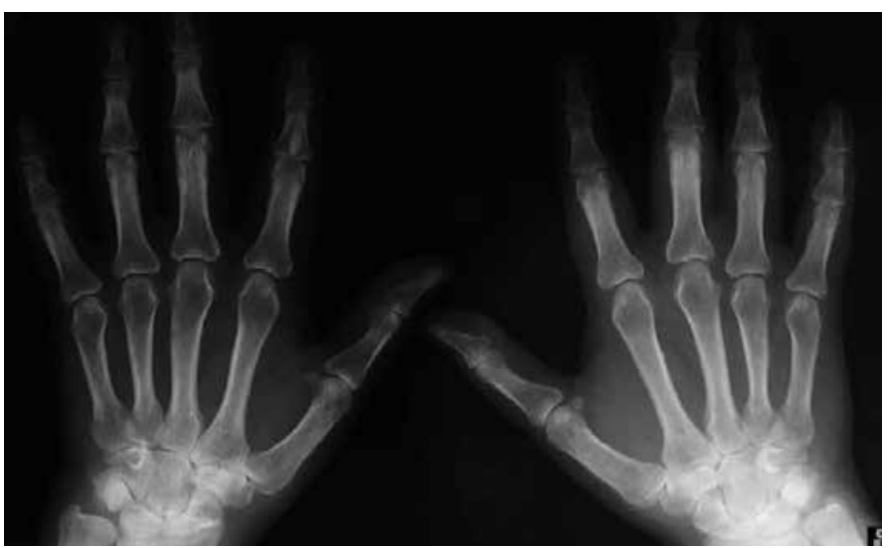

Figure 2. Normal X-ray of both hands

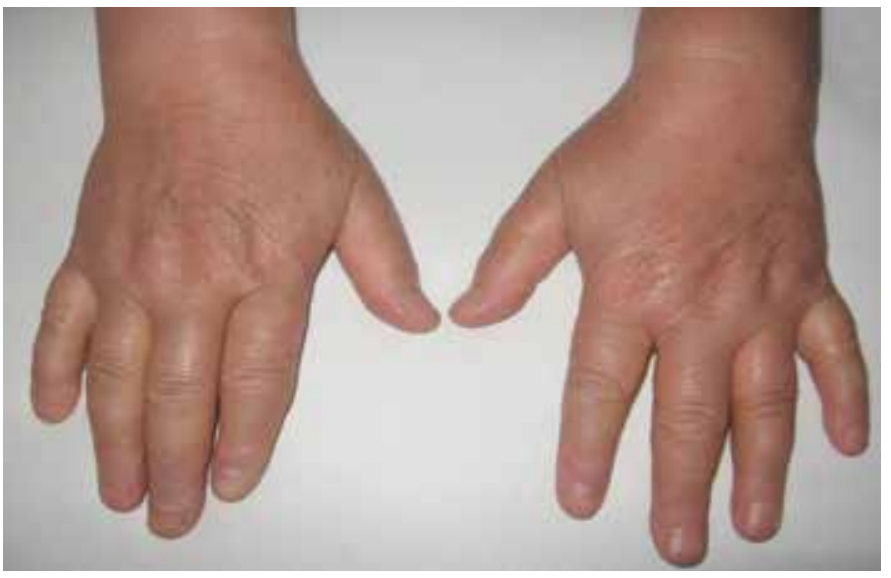

Figure 3. Patient's hands with swollen and tender joints

of a paraneoplastic syndrome. Findings were negative from urine and throat cultures. Liver and renal function tests and urinalysis, peripheral blood smear and levels of antistreptolysin $\mathrm{O}$, markers for hepatitis B and C, anti-HIV antibodies, Brucella and Salmonella, and chest X-ray and abdominal ultrasonography were within normal ranges. Thoracic computed tomography (CT) scans

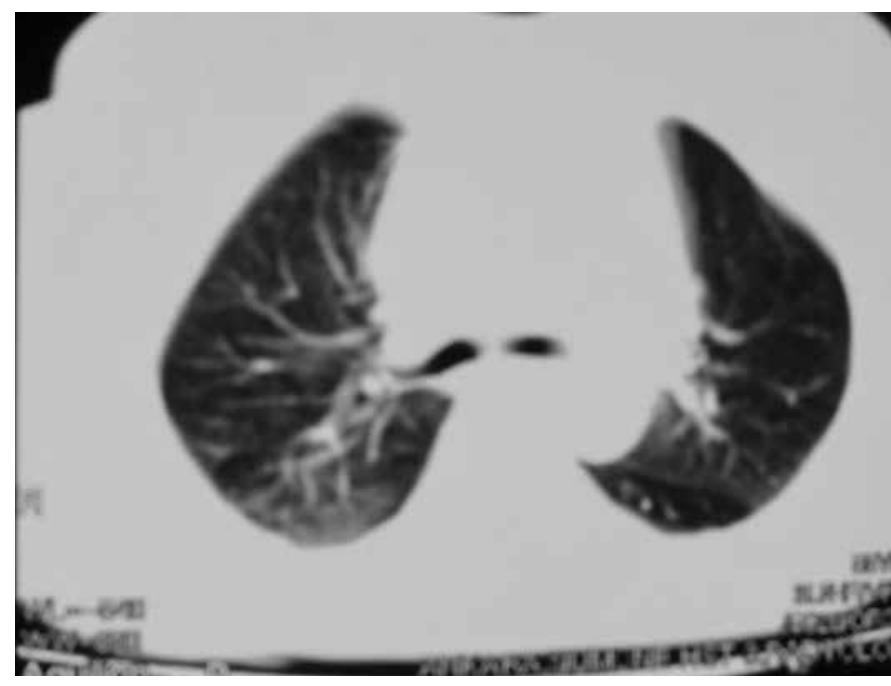

Figure 4. Thoracic computed tomography (CT) scans showing frosted-glass image and fibrotic bands in the lower lobes of both lungs

showed lymphadenopathies located in the subcarinal region and aortopulmonary window accompanied with frosted-glass image and fibrotic bands in the lower lobes of both lungs (Figure 4). Her sputum smear examinations for acid-resistant bacilli and T-spot test were positive. Therefore, she was finally diagnosed with PD; her antirheumatic drugs were stopped, and four-drug antituberculosis therapy was started. At the sixth month visit after discharge, her complaints and clinical symptoms completely disappeared, and her laboratory tests revealed an ESR of $12 \mathrm{~mm} /$ hour and a CRP level of $18.5 \mathrm{mg} / \mathrm{L}$, and parenchymal abnormalities were markedly improved in the control thoracic CT scans. Here we report a case of PD after we obtained the informed consent of the patient.

\section{Discussion}

Poncet's Disease was first described as a type of reactive arthritis in 1897. It more often affects young people and is slightly more common in women. Clinical findings include fever, malaise, and polyarthritis of large joints. The pathogenesis of PD remains to be established; however, it appears to be multifactorial including the activation of CD4+ and CD8+ T cells and hypersensitivity against tuberculosis (TB) proteins (4).

Diagnosis of PD is generally difficult because it starts insidiously, progresses slowly, and has no extra-articular features. The most frequent clinical sign is the change in the range of motions with pain or painless (5). The fact that TB septic monoarthritis, in which Mycobacterium tuberculosis can be isolated from the joint, may complicate TB infection is widely known. On the other hand, active TB may also be complicated by a sterile reactive arthritis called PD, which is less recognized and therefore frequently missed (1).

In literature, numerous papers reported septic monoarthritis with tuberculosis, but there are few reports on established sterile polyarthritis with acute tuberculosis of the lung or urogenital system $(1,2,6)$. PD predominantly occurs in patients with extrapulmonary tuberculosis (7), and the presence of erythema nodosum is one of the important hallmarks of the disease (1), 
although different immunologically-mediated clinical features associate with tuberculosis including erythematous eruption (8), urticaria (9), erythema induratum of Bazin (9), conjunctivitis (8), and oral ulcer (10) have been reported to occur.

Kroot et al. (1) reported two cases, one of which was admitted with relapsing fever for 10 days, muscle weakness, and fatigue. The examination revealed a temperature of $38.8^{\circ} \mathrm{C}$; polyarthritis involving hand joints, ankles, and knees; and erythema nodosum on both lower legs. After 4 weeks, a culture of bronchial mucus revealed Mycobacterium tuberculosis, and therefore, the diagnosis of PD was made. The other patient was admitted because of persistent fever for several months and generalized joint pain. The examination revealed polyarthritis of elbows, wrists, and ankles; dactylitis; tendinitis of both Achilles; lymphadenopathy in the right axilla; and erythema nodosum of both lower legs. The polyarthritis was diagnosed as PD. They reviewed literature and analyzed 50 cases of PD and concluded that no uniform presentation of PD could be determined, but the improvement in clinical features in a few days with antitubercular therapy is typical for the disease.

Ozgul et al. (6) reported human leukocyte antigen (HLA)B27 positive in two cases of urogenital tuberculosis with asymmetric polyarthritis. Smear that were stained with Ehrlich Ziehl Neelsen and that had undergone polymerase chain reaction tests for Mycobacterium tuberculosis of the ejaculate were positive in both cases, despite negative cultures. Therefore, the patients were diagnosed with PD.

Dall et al. (2) mentioned a case where the patient was admitted with pain in the knees, wrists, and elbows; a weight loss of 11 pounds over the preceding 6 months; and a morning stiffness for 30-60 min. Non-steroidal anti-inflammatory agents did not provide relief from the symptoms. The sputum cultures were positive for Mycobacterium tuberculosis.

Bodur et al. (11) reported a case where the patient had chronic arthritis for eight years. She had been admitted to hospital with swollen ankle joint and a mobile, painless mass at her left breast. Synovial biopsies from her ankle joint and left breast were performed. Histopathological examination revealed caseous ve granulomatous infection compatible with tuberculosis. After four months of antituberculosis therapy, her complaints stopped.

Our case represents the difficulty in the diagnosis of PD. Bilateral joint involvement, morning stiffness and elevated levels of ESR, CRP, and RF also raise the possibility of RA. As mentioned in literature, the symptoms in our patient also resolved in a short period of time with antituberculosis therapy.

\section{Conclusion}

PD should be kept in mind in the differential diagnosis of a patient who is under the risk of tuberculosis and who pres- ents with arthritis. Correct and prompt identification of this rare complication of tuberculosis may lead the clinicians to adequately manage the disease.

Informed Consent: Was obtained from the patient who participated in this study.

Author contributions: Concept - E.E.I., D.K.; Design - E.E.I., D.K.; Supervision - D.K., H.B.; Resource - D.K., E.E.I.; Materials - D.K., E.E.I.; Data Collection and/or Processing - E.E.I., H.B.; Analysis and/or Interpretation - D.K., H.B.; Literature Search - E.E.I., D.K.; Writing - D.K., E.E.I.; Critical Reviews - H.B., D.K.; Other - D.K., H.B.

Conflict of Interest: No conflict of interest was declared by the authors.

Financial Disclosure: The authors declared that this study has received no financial support.

\section{References}

1. Kroot EJ, Hazes JM, Colin EM, Dolhain RJ. Poncet's disease: reactive arthritis accompanying tuberculosis. Two case reports and a review of the literature. Rheumatology 2007;46:484-9. [CrossRef]

2. Dall L, Long L, Stanford J. Poncet's disease: tuberculous rheumatism. Rev Infect Dis 1989;11:105-7. [CrossRef]

3. Hameed K, Karim M, Islam N, Gibson T. The diagnosis of Poncet's disease $\mathrm{Br}$ J Rheumatol 1993;32:824-6. [CrossRef]

4. Marker- Hermann E. Septic arthritis, osteomyelitis, gonococcal and syphilitic arthritis. In: Hochberg MC (ed). Rheumatology. 4th edition. Spain: mosby elsevier limited, 2008. p. 1013-45.

5. Davidson PT, Horowitz I. Skeletal tuberculosis. A review with patient presentations and discussion. Am J Med 1970;48:77-84. [CrossRef]

6. Ozgul A, Baylan O, Taskaynatan MA, Kalyon TA. Poncet's disease (tuberculous rheumatism): two case reports and review of the literature. Int J Tuberc Lung Dis 2005;9:822-4.

7. Malik SK, Khatri GK, Deodhar SD.Tuberculous rheumatism (Poncet's disease). J Indian Med Assoc 1977;69:201-2.

8. Wilkinson AG, Roy S. Two cases of Poncet's disease. Tubercle 1984;65:301-3. [CrossRef]

9. Heinemann $C$, Kaatz M, Elsner P. Erythema induratum of Bazin and Poncet's disease -- successful treatment with antitubercular drugs. J Eur Acad Dermatol Venereol 2003;17:334-6. [CrossRef]

10. Perez C, Torroba L, Gonzalez M, Vives R, Guarch R. Unusual presentation of tuberculous rheumatism (Poncet's disease) with oral ulcers and tuberculid. Clin Infect Dis 1998;26:1003-04. [CrossRef]

11. Bodur $\mathrm{H}$, Bodur $\mathrm{H}$, Akinci $\mathrm{E}$, Yilmaz $\mathrm{O}$, Borman $\mathrm{P}$, Tanyolaç $\mathrm{O}$, et al. Osteoarticular tuberculosis. Tuberk Toraks 2003;51:424-31. 